\title{
P I3-03. Neutralizing antibody to HIV-I Env from hyperimmune bovine colostrum as a mucosal microbicide strategy for preventing virus transmission
}

\author{
M Kramski², R Center ${ }^{2}$, G Rawlin ${ }^{1}$, C Siebentritt ${ }^{2}$ and DF Purcell*2
}

Address: ${ }^{1}$ Immuron Ltd, North Melbourne, Australia and ${ }^{2}$ Microbiology and Immunology, University of Melbourne, Parkville, Australia

* Corresponding author

from AIDS Vaccine 2009

Paris, France. 19-22 October 2009

Published: 22 October 2009

Retrovirology 2009, 6(Suppl 3):PI85 doi:I0.I I86/I742-4690-6-S3-PI85

This abstract is available from: http://www.retrovirology.com/content/6/S3/PI85

(c) 2009 Kramski et al; licensee BioMed Central Ltd.

\section{Background}

Newborn mammals derive passive mucosal and systemic immunity against pathogens from the mothers' first milk (colostrum). Bovine colostrum contains antiviral proteins, such as lactoferrin and high levels of maternal IgG, IgM and IgA that are actively concentrated from serum before birth. Immunising cows with HIV-1 Env gp140 oligomers during pregnancy offers a strategy for preparing highly concentrated antibodies (Abs) with HIV-1 neutralizing activity. In this study we develop HIV immune bovine colostrum derived polyclonal antibodies. We examine whether these might match the protective properties of neutralizing monoclonal-Abs.

\section{Methods}

Soluble clade A, B and C Env gp140 oligomers were purified from transduced HeLa and 293 cell supernatant by lentil lectin affinity chromatography and gel filtration. Four cows ( 2 gravid and 2 non-gravid) were vaccinated intramuscularly with $100 \mu \mathrm{g}$ of purified gp140 oligomers in a proprietary immunostimulatory formulation. Two cows were vaccinated with a clade B Env oligomer whereas the other two cows were vaccinated with equal amounts of clade A, B and C Env gp140 oligomers. All cows got two boost vaccinations within 14 weeks.

\section{Results}

All 4 cows seroconverted within 9 weeks post-vaccination with reciprocal endpoint serum IgG titers of up to $1 \times 10^{5}$ for non-gravid and $1.5 \times 10^{2}$ for gravid cows. No increase in the $\mathrm{Ab}$ titre was observed after the second boost vaccination. A panel of different clade A, B and C Env-pseudotyped reporter viruses were produced to test the breadth neutralising activity of the serum-derived bovine Abs that were concentrated into colostrum. Serum, unfractionated colostrum samples and purified colostrum Ig show strong HIV Env binding activity and neutralize HIV Env-pseudotyped reporter viruses.

\section{Conclusion}

Our vaccination strategy yields high titre HIV Env polyclonal antibodies in bovine sera that were concentrated into colostrum. The potential of scale up colostrum Ab production makes this approach potentially feasible for the development of a passive mucosal-administered immunepowered microbicide. 